\title{
Analysis of the independent risk factors of neurologic deficit after thoracolumbar burst fracture
}

\author{
Peifu Tang, Anhua Long, Tao Shi, Licheng Zhang and Lihai Zhang*
}

\begin{abstract}
Background: The objective of this study is to identify the independent risk factors of neurologic deficit after thoracolumbar burst fracture. Traumatic fractures of the thoracolumbar spine are the most common type of spinal column fractures. Many studies have attempted to determine whether neurologic deficit in such fractures is related to spinal canal stenosis or other parameters observed on axial computed tomography. However, this relationship remains controversial.
\end{abstract}

Methods: A review of the clinical data and axial computed tomography (CT) for 105 patients was performed. Neurologic deficit was classified according to the American Spinal Injury Association (ASIA) classification. Various preoperative CT parameters, including vertebral body compression, canal stenosis, sagittal alignment, and fragment reverse, were analyzed using ordinal logistic regression analysis.

Results: Arbeitsgemeinschaft für Osteosynthesefragen (AO) classification, canal volume, transverse canal diameter, median sagittal diameter, Cobb angle, compression ratio of the sagittal diameter, compression ratio of the crosssectional area, and compression ratios of the anterior vertebral height (AVH), middle vertebral height (MVH), and posterior vertebral height (PVH) were significantly associated with severity of nerve injury $(P<0.05)$. However, flip angle and rotation angle of bony fragments were unrelated to severity of nerve damage. Multivariate logistic regression identified $\mathrm{AO}$ classification, compression ratio of median sagittal diameter, anterior vertebral compression ratio, and distance from the posterior margin to the vertebral body above to be independent variables associated with neurologic deficit.

Conclusions: The four $\mathrm{CT}$ parameters most strongly associated with neurologic deficit in thoracolumbar burst fractures are $\mathrm{AO}$ classification, compression ratio of median sagittal diameter, anterior vertebral compression ratio, and distance from the posterior margin to the vertebral body above.

Keywords: Spinal fractures, Spinal cord compression, Neurological examination, Computed tomography, Logistic models

\section{Background}

Traumatic fractures of the thoracolumbar spine, especially of the thoracolumbar junction (T10-L2), are the most common spinal column fractures. High activity and lack of stability make the thoracolumbar spine more prone to fracture. Thoracolumbar burst fracture, defined as a fracture or comminution of both the anterior and middle columns with retropulsion of bony fragments

\footnotetext{
*Correspondence: zhanglihai74@qq.com

Department of Orthopedics, General Hospital of Chinese PLA, No. 28 Fuxing Road, Beijing 100853, People's Republic of China
}

into the spinal canal [1], accounts for approximately 50 to $60 \%$ of all thoracolumbar fractures that cause neurologic deficit $[2,3]$.

Many studies have attempted to determine whether neurologic deficit in such fractures is related to spinal canal stenosis or other parameters observed on axial computed tomography $(\mathrm{CT})$. However, this relationship remains controversial [3-10]. In 1992, Fontijne et al. reported a positive correlation between neurologic deficit and spinal canal stenosis in 139 patients with thoracolumbar burst fracture [4]. However, there was no 
predictive value for the severity of neurologic deficit. Other imaging parameters have been shown in several other studies to be useful in predicting the severity of neurologic deficit after thoracolumbar burst fracture $[11,12]$. In contrast, in 2008, Mohanty et al. found no association between the extent of canal stenosis and the severity of neurologic deficit [9]. Moreover, they found a significant correlation between thoracic spine, rather than L1, and the severity of neurologic deficit.

Most studies have emphasized that spinal canal stenosis and posterior ligamentous complex injury after a fracture lead to neurologic damage [13-16]. However, few reports shed light on the correlation between sagittal alignment, rotation angle of bony fragments, and severity of nerve damage, and no multivariate statistical analysis has considered all prospective indicators related to neurologic damage or found ways to assess neurologic deficit using radiographic parameters. In this study, four radiographic parameters were chosen to assess neurologic deficit after thoracolumbar burst fracture: vertebral body compression, canal stenosis, sagittal alignment, and reverse fragments. The purpose of this study was to identify independent risk factors correlating with neurologic deficit after thoracolumbar burst fracture using a multivariate logistic regression model. Through this study, we would like to analyze some parameters from CT scan that are strongly associated with neurologic deficit, in order to judge prognosis and guide surgery.

\section{Methods}

\section{Patients}

We reviewed the clinical records of patients admitted to our department from January 2009 to December 2011 with trauma-associated, single-segment thoracolumbar burst fracture without posterior longitudinal ligament damage. The inclusion criterion was traumaassociated, single-segment thoracolumbar burst fracture. The exclusion criteria were multi-level vertebral fractures, osteoporosis, cancer metastasis, spondylolisthesis, burst fracture with displacement, ankylosing spondylitis, and degenerative arthritis of the hip or knee. All investigations were carried out in accordance with the ethical guidelines and were approved by the Institutional Ethical Review Committee of Chinese PLA General Hospital (20090121).

\section{Experimental instruments}

A Somatom Sensation Open 40-slice CT scanner (Siemens AG, Erlangen, Germany) was used for preoperative CT scanning. The imaging workstation was from Siemens AG. syngo image-processing software (Siemens AG) was used for image analysis (Fig. 1).

\section{Measurement of imaging parameters}

$\square$ Vertebral body compression. The average of anterior vertebral height, middle vertebral height, and posterior vertebral height $(\mathrm{AVH}, \mathrm{MVH}$, and $\mathrm{PVH}$, respectively) of the vertebral bodies. From top to bottom of the injured vertebra was considered normal height. Compression ratios of $\mathrm{AVH}, \mathrm{MVH}$, and PVH were calculated [17].

$\square$ Canal stenosis. Median sagittal diameter and transverse canal diameter have been used as important indicators for a patient's spinal condition. Thus, in the CT images of the pedicle level, those fracture fragments were obviously visible; the sagittal and transverse canal diameters of the spinal canal were measured.

- Compression ratio of median sagittal diameter. The compression ratio of the median sagittal diameter was calculated using an equation $a=(1-x / y) \times$ $100 \%$. A means compression ratio of median sagittal diameter, where $x$ means median sagittal diameter of the spinal canal at the fracture level and $y$ means normal median sagittal diameter of the spinal canal equal to the average vertebral canal sagittal diameter of the segments from top to bottom [5].

- Compression ratio of canal cross-sectional area. When assessing the degree of compression of the spinal canal, compression ratio of the canal crosssectional area was measured to assess spinal compression after sudden fracture. Average canal cross-sectional area of the segments from top to bottom was considered normal canal cross-sectional area [18].

$\square$ Sagittal alignment.

- Distance from the posterior margin of fractured segment to that of the vertebral body from top to bottom. The distance between the posterior margin of the fractured segment and that of the adjacent segment reflected the length of the posterior longitudinal ligament, which further reflected the injury of the spinal sequence [19].

- Cobb angle. The angle between a line parallel to the superior endplate of the vertebra above the fracture and a line parallel to the inferior endplate of the vertebra below the fracture reportedly has the highest reliability within and between groups [20].

$\square$ Fragment rotation.

- Flip angle of bony fragment in sagittal plane. In the sagittal plane, the flip angle was defined as the angle between a line parallel to the posterior wall of the bony fragment and that of a normal vertebral body from top to bottom [21].

- Rotation angle of bony fragments in the horizontal plane. In the horizontal plane, rotation angle was 


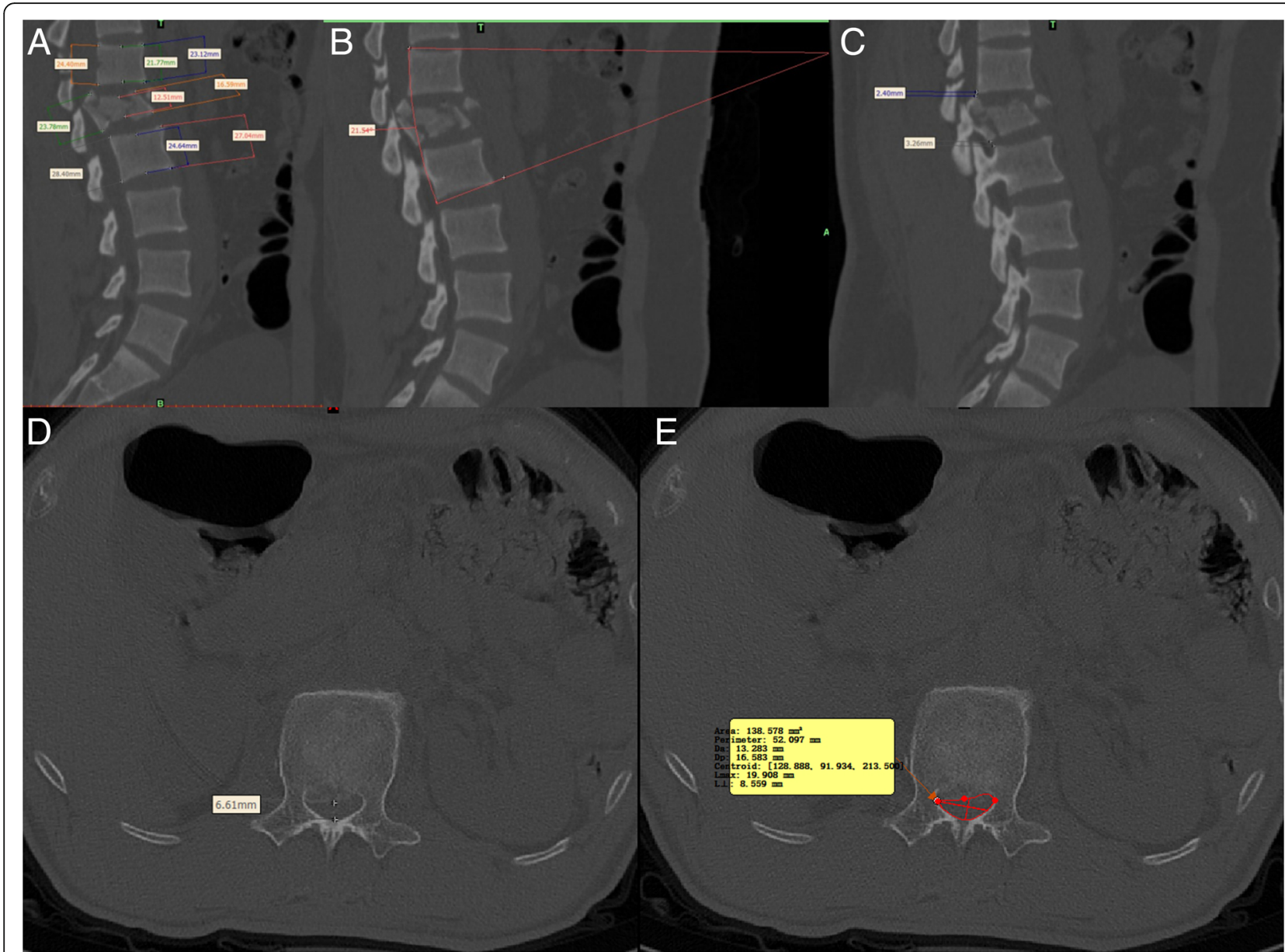

Fig. 1 a: Anterior, median, and posterior vertebral wall heights. b Local kyphosis angle. c Distances of the posterior vertebral edge of the injured vertebrae from the upper and lower adjacent posterior vertebral walls. d Median vertebral canal sagittal diameter. e Cross-sectional area of the vertebral canal

defined as the angle between a line parallel to the posterior wall of the bony fragment and that of a normal vertebral body from top to bottom [22, 23]. $\square$ Arbeitsgemeinschaft für Osteosynthesefragen (AO) classification. The Müller AO classification of fractures was used [24]. A3.1 Incomplete burst fracture: a fracture on the edge of a vertebral body of which the upper and lower endplates are basically intact and there is no intervertebral disc lesion.

This type of fracture is given a score of 1 point. A3.2 Burst split fracture: a vertebral body split fracture with superior and inferior endplate lesions, with no intervertebral disc lesion (2 points). A3.3 Complete burst fracture: comminuted fracture of the vertebral body. Complete shattering of upper and lower endplates and no intervertebral disc lesion (2 points).

\section{Assessing neurologic deficit}

Neurologic deficit was classified according to the American Spinal Injury Association (ASIA) classification [25].

\section{Statistical analysis}

SPSS for Windows, Version 13.0 (SPSS Inc., Chicago, IL, USA), was used for statistical analysis. Normal distributions of continuous-variable data are reported as arithmetic mean \pm standard deviation and non-normal distribution data as median (25th and 75th percentiles). A single-factor ordinal polytomous logistic regression analysis was used to compare differences in parameters between different ASIA grades. All possible parameters underwent multivariate, ordinal, polytomous logistic regression analysis. $P<0.05$ was defined as statistically significant.

\section{Results}

One hundred and thirty-seven patients with thoracolumbar burst fracture were admitted to our service from January 2009 to December 2011, of whom 105 patients (66 were male [62.8\%]; mean age $38.0 \pm 11.8$ years [range, 18-63 years]) met the inclusion criteria and 31 were excluded. Demographic information, including fracture levels and AO and ASIA grade, of the enrolled 
patients is shown in Table 1 . Twenty AO type A patients (47.6\%), 15 AO type B patients (62.5\%), and $32 \mathrm{AO}$ type $C$ patients $(82.1 \%)$ suffered from neurologic deficit. There was a significant positive correlation between $\mathrm{AO}$ classification and severity of nerve injury $(P=0.001)$.

Table 2 shows that CT parameters such as canal volume, transverse canal diameter, median sagittal diameter, compression ratio of sagittal diameter, and cross-sectional area were significantly associated with severity of nerve injury $(P<0.05)$. In other words, the smaller the transverse canal diameter or sagittal diameter, the more serious the nerve injury; the higher the compression ratio of sagittal diameter and cross-sectional area, the more serious the nerve damage. Compression ratios of the anterior, middle, and posterior vertebral heights were also positively correlated with ASIA grade. Thus, there was a significant likelihood of spinal cord compression and nerve damage if compression ratios were high $(P=0.001)$. With regard to sagittal alignment, the smaller the posterior margin distance, the greater the likelihood of nerve damage. However, no significant correlation was observed between the parameters of posterior margin distance and ASIA grade. Cobb angle and severity of nerve injury were positively correlated $(P=0.039)$. Flip angle and rotation angle of bony fragments were unrelated to severity of nerve damage (Table 2).

Table 3 presents the results of multivariate logistic regression analysis of $\mathrm{AO}$ classification and all parameters that may cause nerve damage. We found that $\mathrm{AO}$ fracture classification, compression ratio of median sagittal diameter, anterior vertebral compression ratio, and distance from the posterior margin of the fractured segment to that of the vertebral body above were four

Table 1 Demographics of the thoracolumbar burst fracture patients included in this study

\begin{tabular}{lll}
\hline & Numbers & SD/\% \\
\hline Mean age & 38.0 & 11.8 \\
Male & 66 & $62.8 \%$ \\
Female & 39 & $37.2 \%$ \\
T11 & 10 & $9.5 \%$ \\
T12 & 21 & $20.0 \%$ \\
L1 & 45 & $42.9 \%$ \\
L2 & 29 & $27.6 \%$ \\
AO type A & 42 & $40.0 \%$ \\
AO type B & 24 & $22.9 \%$ \\
AO type C & 39 & $37.1 \%$ \\
ASIA A & 38 & $36.2 \%$ \\
ASIA B & 17 & $16.2 \%$ \\
ASIA C & 14 & $13.3 \%$ \\
ASIA D & 15 & $14.3 \%$ \\
ASIA E & 21 & $20.0 \%$ \\
\hline
\end{tabular}

independent predictors of severity of nerve damage after thoracolumbar burst fracture.

\section{Discussion}

Few reports shed light on the correlation between sagittal alignment, rotation angle of bony fragments, and severity of nerve damage, and no multivariate statistical analysis has considered all prospective indicators related to neurologic damage or found ways to assess neurologic deficit using radiographic parameters. In this study, four radiographic parameters were chosen to assess neurologic deficit after thoracolumbar burst fracture: vertebral body compression, canal stenosis, sagittal alignment, and reverse fragments. The purpose of this study was to identify independent risk factors correlating with neurologic deficit after thoracolumbar burst fracture using a multivariate logistic regression model.

Denis investigated 412 patients with spinal fractures and proposed the spinal three-column theory to create a more detailed discussion of fractures using CT images. As defined using this theory, bony fragments from thoracolumbar burst fractures are retropulsed with greater intensity into the spinal canal.

Many studies focus on the complications including neurological status after thoracolumbar fractures [13-16]. Andreas reported preoperative neurological deficits (American Spinal Injury Association (ASIS) Classification), 15 ASIS B deficits and 8 ASIS C deficits in 25 patients with thoracolumbar fractures (T11-L2), with Denis classifications. Palvos reported preoperative neurological deficits (ASIS classification), 75 ASIS E deficits and 25 ASIS (A-D) deficits in 100 patients of thoracolumbar fractures (T11-L2), with AO-Magerl classification. Kalliopi discussed and reported that integrity of the posterior ligament has some relationship with neurological deficits. However, all the discussions focus on the main classification and not on the specific parameters.

Our results indicate that four radiographic parameters correspond to the severity of nerve damage after thoracolumbar burst fracture: AO classification of fracture, compression ratio of median sagittal diameter, anterior vertebral compression ratio, and the distance from the posterior margin to that of the vertebral body above. Most previous studies considered canal stenosis after spinal fracture to be the main indicator of nerve damage. Spinal cord compression was assessed by measuring the sagittal diameter of the spinal canal on axial CT. Some studies consider canal volume to reflect nerve damage, but this is still controversial. Rasmussen et al. [15] found that the cross-sectional area of the vertebral canal was a better parameter by which to assess spinal compression. Compared with compression ratio of sagittal diameter, the compression ratio of cross-sectional area had a stronger correlation with neurological function. However, cross-sectional 
Table 2 Comparison between computerized tomographic parameters and ASIA classification

\begin{tabular}{|c|c|c|c|c|c|c|c|c|c|c|c|}
\hline \multirow[b]{2}{*}{ AO classification } & \multicolumn{2}{|c|}{$\begin{array}{l}\text { ASIA A } \\
38 \text { cases }\end{array}$} & \multicolumn{2}{|c|}{$\begin{array}{l}\text { ASIA B } \\
17 \text { cases }\end{array}$} & \multicolumn{2}{|c|}{$\begin{array}{l}\text { ASIA C } \\
14 \text { cases }\end{array}$} & \multicolumn{2}{|c|}{$\begin{array}{l}\text { ASIA D } \\
15 \text { cases }\end{array}$} & \multicolumn{2}{|c|}{$\begin{array}{l}\text { ASIA E } \\
21 \text { cases }\end{array}$} & \multirow{2}{*}{$\frac{P \text { value }}{0.001}$} \\
\hline & 22 & $58 \%$ & 8 & $47 \%$ & 4 & $29 \%$ & 4 & $27 \%$ & 4 & $19.0 \%$ & \\
\hline Transverse canal diameter & 25.48 & $(22.65,27.63)$ & 26.54 & $(23.84,28.16)$ & 26.24 & $(23.44,28.22)$ & 24.19 & $(20.64,28.82)$ & 22.68 & $(0.01,27.65)$ & 0.003 \\
\hline Median sagittal diameter & 9.76 & $(7.47,13.94)$ & 11.02 & $(7.29,15.27)$ & 12.03 & $(7.25,14.01)$ & 8.95 & $(6.17,11.66)$ & 6.27 & $(0.01,8.76)$ & 0.001 \\
\hline Compression ratio of median sagittal diameter & 0.365 & $(0.07,0.502)$ & 0.308 & $(0.031,0.504)$ & 0.367 & $(0.084,0.574)$ & 0.476 & $(0.288,0.788)$ & 0.600 & $(0.434,1.000)$ & 0.001 \\
\hline Compression ratio of canal cross-sectional area & 0.256 & $(0.045,0.435)$ & 0.274 & $(0.082,0.445)$ & 0.171 & $(0.046,0.565)$ & 0.218 & $(0.106,0.44)$ & 0.386 & $(0.246,0.839)$ & 0.004 \\
\hline Compression ratios of $\mathrm{AVH}$ & 0.146 & $(0.107,0.294)$ & 0.190 & $(0.138,0.276)$ & 0.259 & $(0.147,0.309)$ & 0.386 & $(0.254,0.47)$ & 0.363 & $(0.256,0.454)$ & 0.001 \\
\hline Compression ratios of $\mathrm{MVH}$ & 0.104 & $(0.068,0.185)$ & 0.097 & $(0.056,0.17)$ & 0.158 & $(0.078,0.225)$ & 0.199 & $(0.075,0.277)$ & 0.248 & $(0.105,0.343)$ & 0.001 \\
\hline Compression ratios of $\mathrm{PVH}$ & 0.191 & $(0.112,0.281)$ & 0.181 & $(0.107,0.247)$ & 0.259 & $(0.189,0.341)$ & 0.276 & $(0.119,0.387)$ & 0.286 & $(0.181,0.392)$ & 0.005 \\
\hline The distance of posterior margin with upper vertebra & 4.95 & $(4.31,6.12)$ & 5.02 & $(3.64,5.92)$ & 4.76 & $(3.51,5.86)$ & 5.08 & $(4.02,6.49)$ & 4.65 & $(3.97,5.15)$ & 0.200 \\
\hline The distance of posterior margin with below vertebra & 3.98 & $(3.27,4.66)$ & 4.29 & $(3.25,5.24)$ & 3.86 & $(3.21,4.64)$ & 3.59 & $(2.76,4.23)$ & 3.66 & $(3.36,4.89)$ & 0.302 \\
\hline Cobb angle & 10.3 & $(8.6,14.0)$ & 11.3 & $(2.9,21.0)$ & 10.7 & $(7.1,17.9)$ & 12.9 & $(10,18.7)$ & 16.2 & $(9.4,22.8)$ & 0.039 \\
\hline Flip angle & 16.44 & $(10.24,31.59)$ & 15.90 & $(7.92,28.3)$ & 32.26 & $(15.47,43.6)$ & 28.26 & $(10.9,42.31)$ & 21.61 & $(10.54,37.64)$ & 0.189 \\
\hline Rotation angle & 0.10 & $(0.1,4.75)$ & 2.95 & $(0.1,3.94)$ & 3.21 & $(0.1,5.8)$ & 4.30 & $(1.96,5.63)$ & 2.52 & $(0.1,5.46)$ & 0.366 \\
\hline
\end{tabular}


Table 3 Multivariate analysis using ordinal logistic regression models to evaluate the independent risk factors of neurologic deficit after thoracolumbar burst fracture

\begin{tabular}{|c|c|c|c|c|}
\hline & OR & $95 \% C$ & & $P$ value \\
\hline Transverse canal diameter & 0.96 & 0.9 & 1.02 & 0.203 \\
\hline Median sagittal diameter & 1.06 & 0.89 & 1.27 & 0.513 \\
\hline Compression ratio of median sagittal diameter & 26.1 & 1 & 684.71 & 0.050 \\
\hline Compression ratio of canal cross-sectional area & 0.94 & 0.05 & 16.59 & 0.966 \\
\hline Compression ratios of $\mathrm{AVH}$ & 533.79 & 10.09 & 28226.03 & 0.002 \\
\hline Compression ratios of $\mathrm{MVH}$ & 27.03 & 0.2 & 3662.86 & 0.188 \\
\hline Compression ratios of $\mathrm{PVH}$ & 0.01 & 0 & 3.03 & 0.118 \\
\hline The distance of posterior margin with upper vertebra & 0.62 & 0.43 & 0.9 & 0.013 \\
\hline The distance of posterior margin with below vertebra & 1.08 & 0.72 & 1.63 & 0.712 \\
\hline Cobb angle & 1.01 & 0.94 & 1.06 & 0.952 \\
\hline Flip angle & 0.98 & 0.95 & 1.01 & 0.091 \\
\hline Rotation angle & 1.03 & 0.99 & 1.07 & 0.196 \\
\hline AO classification type A & 0.18 & 0.06 & 0.54 & 0.002 \\
\hline AO classification type B & 0.31 & 0.1 & 0.96 & 0.042 \\
\hline
\end{tabular}

area of the spinal canal varies with sex and ethnicity. When assessing compression of the spinal canal after fracture, use of the compression ratio of the cross-sectional area may effectively avoid bias associated with sex and ethnicity. In our study, compression ratio of sagittal diameter and cross-sectional area were associated with nerve injury. However, multivariate analysis showed that compression ratio of sagittal diameter had a stronger correlation with the severity of nerve damage than did the compression ratio of the cross-sectional area.

Rotation of retropulsion bony fragments in the vertebral canal as well as change in canal volume was taken into account. Thoracolumbar burst fractures create bony fragments of the posterior wall, which are retropulsed into the vertebral canal. Guerra et al. [26] found retropulsion and rotation of bony fragments, a phenomenon that was later confirmed on CT. Flipping of bone fractures may reflect the severity of injury and may therefore predict nerve damage. However, according to our study, flip angle and rotation angle of bony fragments were unrelated to severity of nerve damage.

Changes in vertebral height may reflect severity of canal stenosis and nerve compression. Parameters including the compression ratios of the $\mathrm{AVH}, \mathrm{MVH}$, and $\mathrm{PVH}$; vertebral flip angle; and cross-diagonal angle are widely used to measure changes in vertebral height [11]. It is also generally believed that surgery may be indicated for a vertebral compression ratio of $>50 \%$ after thoracolumbar burst fracture [14]. AVH, MVH, and PVH compression ratios may accurately reflect the severity of nerve injury after fracture, with the strongest correlation being between the ratio of $\mathrm{PVH}$ and the severity of nerve injury.
Changes in the overall sequence of vertebrae often occur after thoracolumbar burst fracture, with disruption of the relative positions of the upper and lower vertebrae. Therefore, we measured both Cobb angle and the distance between the posterior margin of the fracture segment and the neighboring segment to assess changes in vertebral sequence. The results showed that refined Cobb angle was promising in its ability to predict the severity of the fracture and nerve damage. Cobb angle was originally used to describe the integrity of the posterior longitudinal ligament and deformity in the coronal plane. It was also used to assess degree of spinal vertebral kyphosis [27]. However, no report to date has shown a relationship between Cobb angle and nerve injury after thoracolumbar burst fracture.

A number of previous studies considered canal volume as well as vertebral height to be predictors of nerve injury after thoracolumbar burst fracture [4, 7-10]. In the present study, we performed statistical analysis of radiographic parameters of axial CT, such as compression of canal cross-sectional area and vertebral height, to find the parameters most closely associated with nerve damage and found spinal canal volume, vertebral height, and sagittal alignment to be the best predictors. AO classification was also a good predictor of the degree of nerve damage.

There are some limitations in this study. Firstly, all the measurements were made by human beings; there must be some measurement bias. Additionally, the patient's number is limited because of the number of fractures. Lastly, the CT scan can only reflect the situation when the patient did the test; the mechanism of injury and the injury moment of fracture should be considered. A 
multicenter study and more accuracy measurements or $3 \mathrm{D}$ measurements in the spinal canal will better the design for further studies.

\section{Conclusions}

Our study indicated that the four CT parameters with the strongest associations with neurologic deficit than that of other parameters in thoracolumbar burst fractures were $\mathrm{AO}$ classification of fracture, compression ratio of median sagittal diameter, anterior vertebral compression ratio, and distance from the posterior margin to the vertebral body above.

\section{Abbreviations}

AO: Arbeitsgemeinschaft für Osteosynthesefragen; ASIA: The American Spinal Injury Association; AVH: Anterior vertebral height; CT: Computed tomography; MVH: Middle vertebral height; PVH: Posterior vertebral height

\section{Acknowledgements}

Not applicable.

\section{Funding}

This work was supported by a grant from the National High Technology Research and Development Program of China (863 Program) (No. SS2012AA041604).

\section{Availability of data and materials}

Not applicable.

\section{Authors' contributions}

PT contributed to the article writing and data collection. AL contributed to the article writing and data statistics. TS contributed to the data collection and article writing. LZ contributed to the article writing and project design. All authors read and approved the final manuscript.

\section{Competing interests}

The authors declare that they have no competing interests.

\section{Consent for publication}

Not applicable.

\section{Ethics approval and consent to participate}

All investigations were carried out in accordance with the ethical guidelines and were approved by the Institutional Ethical Review Committee of Chinese PLA General Hospital (20090121).

Received: 5 June 2016 Accepted: 17 September 2016

Published online: 24 October 2016

\section{References}

1. Denis $F$. The three column spine and its significance in the classification of acute thoracolumbar spinal injuries. Spine. 1983;8(8):817-31.

2. Trafton PG, Boyd Jr CA. Computed tomography of thoracic and lumbar spine injuries. J Trauma. 1984:24(6):506-15.

3. Vaccaro AR, Nachwalter RS, Klein GR, Sewards JM, Albert TJ, Garfin SR. The significance of thoracolumbar spinal canal size in spinal cord injury patients. Spine. 2001;26(4):371-6.

4. Fontijne WP, de Klerk LW, Braakman R, Stijnen T, Tanghe HL, Steenbeek R, van Linge B. CT scan prediction of neurological deficit in thoracolumbar burst fractures. J Bone Joint Surg Br Vol. 1992;74(5):683-5.

5. HASHIMOTO T, KANEDA K, ABUMI K. Relationship between traumatic spinal canal stenosis and neurologic deficits in thoracolumbar burst fractures. Spine. 1988;13(11):1268-72

6. Kim NH, Lee HM, Chun IM. Neurologic injury and recovery in patients with burst fracture of the thoracolumbar spine. Spine. 1999;24(3):290-3. discussion 4.
7. Meves R, Avanzi O. Correlation among canal compromise, neurologic deficit, and injury severity in thoracolumbar burst fractures. Spine. 2006:31(18):2137-41.

8. Meves R, Avanzi O. Correlation between neurological deficit and spinal canal compromise in 198 patients with thoracolumbar and lumbar fractures. Spine. 2005;30(7):787-91.

9. Mohanty S, Bhat N, Abraham R, Keerthi Cl. Neurological deficit and canal compromise in thoracolumbar and lumbar burst fractures. J Orthop Surg. 2008;16(1):20-3.

10. Yugue I, Aono K, Shiba K, Ueta T, Maeda T, Mori E, Kawano O. Analysis of the risk factors for severity of neurologic status in 216 patients with thoracolumbar and lumbar burst fractures. Spine. 2011;36(19):1563-9.

11. Isomi T, Panjabi MM, Kato Y, Wang JL. Radiographic parameters for evaluating the neurological spaces in experimental thoracolumbar burst fractures. J Spinal Disord. 2000;13(5):404-11.

12. Keynan O, Fisher CG, Vaccaro A, Fehlings MG, Oner FC, Dietz J, Kwon B, Rampersaud R, Bono C, France J, Dvorak M. Radiographic measurement parameters in thoracolumbar fractures: a systematic review and consensus statement of the spine trauma study group. Spine. 2006;31(5):E156-65.

13. Vaccaro AR, Rihn JA, Saravanja D, Anderson DG, Hilibrand AS, Albert TJ, Fehlings MG, Morrison W, Flanders AE, France JC, Arnold P, Anderson PA, Friel B, Malfair D, Street J, Kwon B, Paquette S, Boyd M, Dvorak MF, Fisher C. Injury of the posterior ligamentous complex of the thoracolumbar spine: a prospective evaluation of the diagnostic accuracy of magnetic resonance imaging. Spine. 2009;34(23):E841-7.

14. Mavrogenis A, Tsibidakis $H$, Papagelopoulos P, Antonopoulos D, Papathanasiou J, Korres D, Pneumaticos S. Posterior transpedicular decompression for thoracolumbar burst fractures. Folia Med (Plovdiv). 2010;52(4):39-47

15. Alpantaki K, Bano A, Pasku D, Mavrogenis AF, Papagelopoulos PJ, Sapkas GS, Korres DS, Katonis P. Thoracolumbar burst fractures: a systematic review of management. Orthopedics. 2010;33(6):422-9.

16. Katonis P, Pasku D, Alpantaki K, Mavrogenis AF, Korres DS, Sapkas GS, Papagelopoulos PJ. Combination of the AO-Magerl and load-sharing classifications for the management of thoracolumbar burst fractures. Orthopedics. 2010;33(3). doi:10.3928/01477447-20100129-07. Epub 2010 Mar 10.

17. Willen J, Anderson J, Toomoka K, Singer K. The natural history of burst fractures at the thoracolumbar junction. J Spinal Disord Tech. 1990;3(1):39-46.

18. RASMUSSEN PA, RABIN MH, MANN DC, PERL JR, LORENZ MA, VRBOS LA. Reduced transverse spinal area secondary to burst fractures: is there a relationship to neurologic injury? J Neurotrauma. 1994;11(6):711-20.

19. Leferink VJ, Nijboer JM, Zimmerman KW, Veldhuis EF, ten Vergert EM, ten Duis HJ. Burst fractures of the thoracolumbar spine: changes of the spinal canal during operative treatment and follow-up. Eur Spine J. 2003;12(3):255-60.

20. Bridwell KH, Kuklo TR, Lewis SJ, Sweet FA, Lenke LG, Baldus C. String test measurement to assess the effect of spinal deformity correction on spinal canal length. Spine. 2001;26(18):2013-9.

21. Verlaan JJ, van de Kraats EB, van Walsum T, Dhert WJ, Oner FC, Niessen WJ. Three-dimensional rotational $\mathrm{X}$-ray imaging for spine surgery: a quantitative validation study comparing reconstructed images with corresponding anatomical sections. Spine. 2005;30(5):556-61.

22. Beck M, Mittlmeier T, Gierer P, Rotter R, Harms C, Gradl G. Which is the ideal point of time to perform intraoperative $3 \mathrm{D}$ imaging in dorsal stabilisation of thoracolumbar spine fractures? A matched pair analysis. Injury. 2010;41(10):996-1001.

23. Hott JS, Papadopoulos SM, Theodore N, Dickman CA, Sonntag VK. Intraoperative Iso-C C-arm navigation in cervical spinal surgery: review of the first 52 cases. Spine. 2004;29(24):2856-60.

24. Magerl F, Aebi M, Gertzbein SD, Harms J, Nazarian S. A comprehensive classification of thoracic and lumbar injuries. Eur Spine J. 1994;3(4):184-201.

25. Maynard Jr FM, Bracken MB, Creasey G, Ditunno Jr JF, Donovan WH, Ducker TB, Garber SL, Marino RJ, Stover SL, Tator CH, Waters RL, Wilberger JE, Young W. International Standards for Neurological and Functional Classification of Spinal Cord Injury. American Spinal Injury Association. Spinal cord May. 1997;35(5):266-74.

26. Guerra Jr J, Garfin SR, Resnick D. Vertebral burst fractures: CT analysis of the retropulsed fragment. Radiology. 1984;153(3):769-72.

27. Cobb J. The American Academy of Orthopedic Surgeons Instructional Course Lectures. Ann Arbor: Edwards; 1948. 